\title{
Géologie du Liban vue par un géotechnicien. Impact sur les modes de soutènement à Beyrouth
}

\section{y. KAZAN}

Université libanaise Faculté de Génie

BP 90

1780 Jdeidet el Maten Liban

ykazan@ul.edu.lb
Afin de rapprocher les géologues et les ingénieurs civils dans le domaine de la géothechnique au Liban, l'auteur, un géotechnicien, s'efforce de considérer les formations géologiques libanaises sous une vision mécanique. Ainsi, les traits communs dans les caractéristiques géotechniques des différentes formations sont soulignés. Un compromis est fait entre la classification chronologique et minérologique des géologues et les approches des mécaniciens des sols et des roches. Les «travaux géotechniques » traités dans cet article sont les structures de soutènement provisoire conçues et exécutées lors des travaux de terrassement en zone urbaines. Une classifiction locale des modes de soutènement est proposée. Des réflexions sur les procédures géotechniques adoptées dans les villes libanaises sont données. Des critiques sur la méthodologie courante des travaux géotechniques sont formulées.

Mots-clés : géologie, formations sédimentaires, soutènement, géotechnique, grès, Crétacé, terrassement, paramètres mécaniques.

\section{Geological formations of Lebanon as viewed by a geotechnical engineer}

In order to put together Lebanese geologists and civil engineers on geotechnical issues, the author, a geotechnical engineer, examines the Lebanese geological formations in a mechanical perspective. In this way, similarities in the mechanical characteristics of each formation are outlined. A compromise is made between the chronological and mineral classification of the geologists and the mechanical procedures of the geotechnical engineers.

The geotechnical works considered in this paper are the retaining systems provisionally used in excavation in urban areas. This article proposes a local classification of retaining procedures depending on the geological context. The article also includes some remarks on geotechnical procedures utilized in Lebanese cities as well as a discussion on current methodology of geotechnical works.

Key-words: geological formations, sedimentary rock, retaining systems, geotechnics, excavations. 


\section{Introduction}

Bien que de grands projets de travaux publics (barrages, tunnels...) ne soient pas encore exécutés au Liban, les travaux géotechniques sont bien développés dans les soutènements provisoires, dans le cadre de terrassement des excavations. En effet, après une période de quinze ans de guerre, plusieurs projets géotechniques ont été entrepris, durant la dernière décennie, dans les villes libanaises, notamment dans Beyrouth. Les règlements d'urbanisme, récemment mis en vigueur, ont imposé aux entrepreneurs de prévoir des excavations dépassant les quinze mètres de profondeur pour contenir un nombre de six sous-sols en moyenne. Ces bâtiments sont parfois construits à proximité d'édifices en maçonnerie ou d'anciennes habitations superficielles, construites selon les normes des années passées.

Les nombreux projets réalisés ont été conçus et exécutés simultanément avec les techniques variées des différents pays développés (France, Allemagne, Canada...). Nous avons pu voir, à titre d'exemple, les procédures de parois moulées, fréquentes dans les formations molles et lâches, côtoyer les pieux espacés, plutôt exécutés dans les formations imperméables, et les panneaux horizontaux en béton armé dont l'utilisation est justifiée dans les formations cohésives. Durant les années 90, une dizaine de modes de soutènement a été utilisée. Plusieurs critiques ont été formulées sur la façon dont une centaine de projets ont été conduits. Certains soutènements sont surdimensionnés, alors que des incidents ont été enregistrés dans d'autres projets. Une révision objective s'avère donc indispensable. Un examen sommaire à ce stade semble donc nécessaire.

L'analyse exhaustive de ces problèmes doit porter sur plusieurs facteurs : géologiques et mécaniques, techniques et économiques, juridiques et moraux et, peut-être, politiques et culturels. Ne pouvant pas traiter tous ces problèmes dans un mème contexte, nous abordons le sujet dans cet article de la façon suivante : - traiter l'aspect géologique en décrivant la géologie libanaise, assez variée pour une petite superficie, qui trouve ses extensions à Beyrouth et dans les régions alentour:

- souligner et analyser les caractéristiques mécaniques et géotechniques de ces formations ;

- décrire et critiquer les approches adoptées en mécanique des sols et des roches pour caractériser les massifs et présenter sommairement les différents types des structures géotechniques.

En sélectionnant certains facteurs, nous ne minimisons pas l'influence d'autres paramètres : les circonstances du projet, les moyens existants (techniques, scientifiques et financiers), les coutumes et les habitudes traditionnelles. Nous espérons que ces aspects seront développés ouvertement par d'autres rédacteurs en vue d'améliorer les pratiques géotechniques et de progresser à l'échelle technique et scientifique. Nous considérons que ces facteurs seront communs à d'autres villes en cours de changement.

\section{Géologie libanaise}

\section{1}

\section{Historique des travaux géologiques}

Les formations géologiques libanaises ont commencé à être étudiées scientifiquement à partir de la deuxiême moitié du XIX $X^{\varepsilon}$ siècle. Outre les corps d'expéditions français, des scientifiques allemands s'y sont intéressés et ont laissé des traces dans ce domaine (Farras, 1878 ; Kober, 1915). Toutefois, les documents utiles et fiables ont pu être réalisés par les chercheurs français dans la première moitié du XX $X^{c}$ siècle. Les cartes et les renseignements géologiques sur le Liban sont d'ailleurs associés au nom de Louis Dubertret, chef de la Section géologique de la Délégation générale de France au Levant. Suite au même élan, certaines cartes ont pu être publiées au milieu du siècle dernier par le ministère des Travaux publics de la République libanaise indépendante. Les besoins hydrauliques de la population libanaise ont engendré quelques travaux modestes dans l'hydrogéologie (Abd-el-Al, 1949). Enfin, Tinitiative individuelle de quelques géologues académiques contemporains a contribué à l'apparition de quelques publications récentes (Khawlie \& Ghannam, 1987).

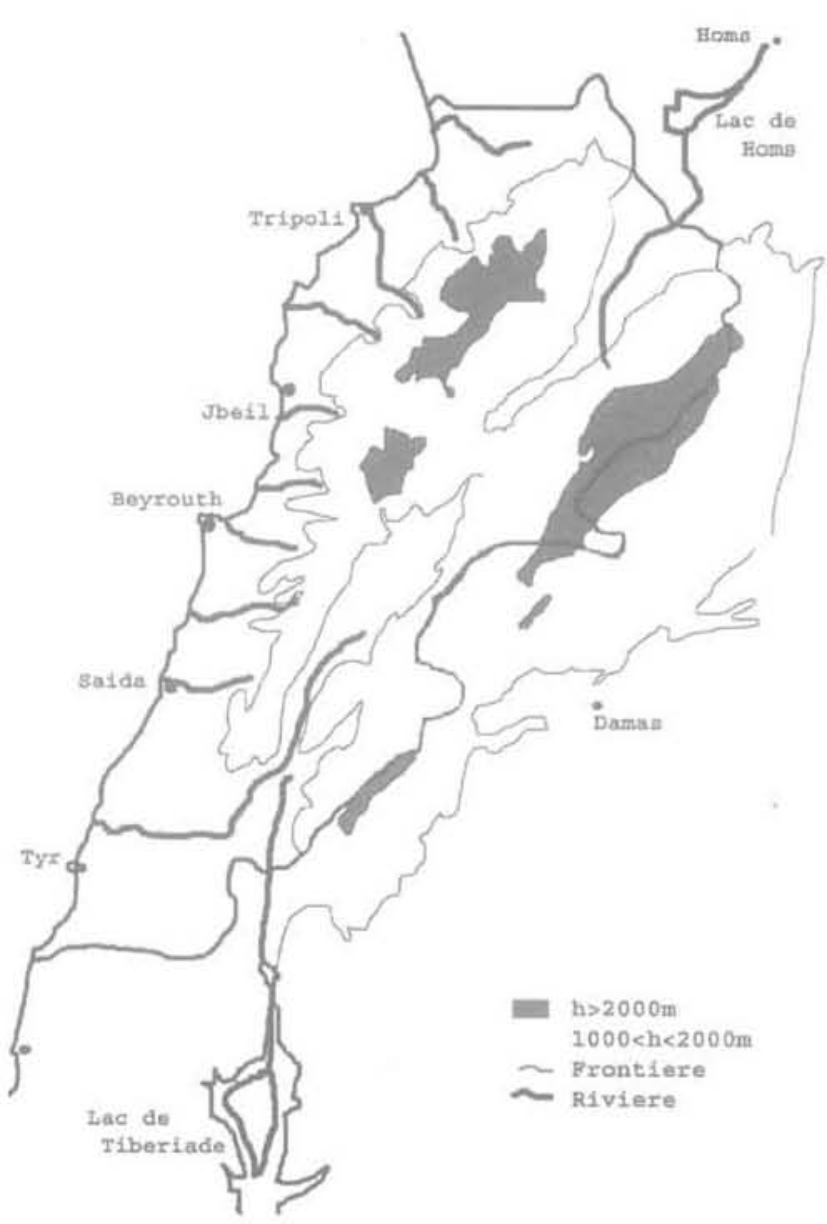

Fị.1 Schéma orographique du Liban. 


\section{Orographie géologique du Liban}

Les traits majeurs du relief du Liban sont deux longs massifs séparés par un sillon médian (plaine de la Bekaa) de 1000 mètres d'altitude environ. Ce complexe orographique, de $170 \mathrm{~km}$ de longueur, est orienté SSW-NNE. Les sommets de ces montagnes culminent à 3000 mètres de hauteur. La population se concentre actuellement dans les régions relativement basses (moins de 1000 mètres). La zone la plus peuplée reste la côte, où plusieurs villes s'étendent sur l'Est de la Méditerranée.

Une douzaine de rivières côtières se dirigent vers l'ouest pour atteindre leurs estuaires méditerranéens. Trois autres rivières enviées, classées intérieures, tracent leurs lits dans la plaine de la Bekaa (Fig. 1). Les cours de ces rivières, ainsi que la plage méditerranéenne, ont permis aux formations quaternaires de trouver une place entre les formations du Jurassique et du Crétacé de l'ère secondaire, et celles du Nummulitique et du Néogène de l'ère tertiaire.

\section{Formations géologiques}

La quasi-totalité des formations géologiques de la superficie libanaise est sédimentaire. Ces formations remontent à l'âge jurassique de l'ère secondaire. Les roches calcaires et les grès sont les deux formations les plus abondantes. Le volcanisme basaltique est erratiquement présent dans certains endroits du pays, le nord en particulier, à proximité de la région syrienne de Homs. Il est toutefois tardif et n'apparait qu'à partir du Miocène. Ses voies d'ascension étaient des failles, vieilles et nouvelles.

Les tableaux I et II regroupent les différentes formations existantes au Liban. Cette classification est basée sur le langage et les expressions utilisées par les géologues depuis des années. Le calcaire est présent à plusieurs stades de formations, dure (Jurassique) et tendre (Turonien). Le grès de base du Crétacé est l'une des formations spécifique du Liban.

La figure 2 montre les formations géologiques à Beyrouth et dans les régions à còté. Notons bien que cette carte géologique montre les formations quaternaires occupant une bonne partie de la capitale en sur-

TABLEAU Formations sédimentaires de l'ère secondaire au Liban décrites par les géologues.

\begin{tabular}{|c|c|c|c|c|}
\hline \multirow{6}{*}{$\begin{array}{l}\text { C } \\
\text { R } \\
\text { E } \\
\text { T } \\
\text { A } \\
\text { C } \\
\text { E }\end{array}$} & Sup. & Sénonien & $c_{6}$ & $\begin{array}{l}\text { Marnes blanches crayeuses. Contenant parfois des nodules } \\
\text { phosphatés (brèches ou graviers) noyés dans un fond } \\
\text { de glauconie. }\end{array}$ \\
\hline & \multirow{3}{*}{ Moyen } & Turonien & $c_{5}$ & $\begin{array}{l}\text { Marno-calcaires, se fond parfois lithologiquement avec } \mathrm{c}_{4} \\
\text { Marnes claires finement et régulièrement litées, chargées de lits } \\
\text { serrès de rognons de silex disposés en stratifications. } \\
\text { Brèches grossières quí passent à du calcaire compact en masses } \\
\text { informes, puis à des marnes calcaires à lits de rognons de silex } \\
\text { très serrés. }\end{array}$ \\
\hline & & Cénomanien & $c_{4}$ & $\begin{array}{l}\text { Alternances de bandes marno-calcaires et de bancs calcaires } \\
\text { finement lités. Silex et géodes quartziques en chou-fleur sont } \\
\text { associés aux marno-calcaires. }\end{array}$ \\
\hline & & Albien & $c_{3}$ & $\begin{array}{l}\text { Grés à oolithes ferrugineux (parfois basaltiques), Alternance } \\
\text { de bancs calcaires et de marne verte. Trés fossilifëres. }\end{array}$ \\
\hline & \multirow[t]{2}{*}{ Inf. } & Aptien & $c_{2}$ & $\begin{array}{l}\text { Alternance du grès, du grès argileux, de la marne et des bancs } \\
\text { calcaires fins (oolithiques ou détritiques). }\end{array}$ \\
\hline & & Grès de base & $\mathrm{c} 1$ & Grès quartziques stratifiés. \\
\hline \multirow{8}{*}{$\begin{array}{l}\text { J } \\
\text { U } \\
\text { R } \\
\text { A } \\
\text { S } \\
\text { S } \\
\text { I } \\
\text { Q } \\
\text { U } \\
\text { E }\end{array}$} & \multirow{5}{*}{ Sup. } & Portlandien & $j_{7}$ & $\begin{array}{l}\text { Calcaire et dolomitique à silex, alternance de calcaire oolithique } \\
\text { ocre et de marnes. }\end{array}$ \\
\hline & & Kimmèridigien & $i_{0}$ & Calcaire massif, brun, marneux, parfois oolithique. \\
\hline & & Lusitanien & $f_{S}$ & $\begin{array}{l}\text { Marne argileuse grise, avec concrétions d'hématite. } \\
\text { Calcaire jaunâtre avec intercalations marneuses. } \\
\text { Calcaire blanc, en bancs. }\end{array}$ \\
\hline & & Oxfordien & $j_{4}$ & $\begin{array}{l}\text { Marnes claires ou bleutées à petits céphalopodes pyriteux ou } \\
\text { limoniteux. } \\
\text { Marne calcaire grise avec bancs de rognons calcaires dans la } \\
\text { partie supérieure. }\end{array}$ \\
\hline & & Callovien & $j_{4}$ & Calcaire gris avec intercalations de feuillets marno-calcaires. \\
\hline & \multirow[b]{2}{*}{ Moyen } & Bathonien & $\mathrm{j}_{4}$ & Dolomies noires. Calcaire dolomitique. \\
\hline & & Bajocien & $\begin{array}{l}j_{2} \\
\text { et } \\
j_{3}\end{array}$ & $\begin{array}{l}\text { Calcaire brun, à nodules de schizophytes et quelques } \\
\text { intercalations de minces lits marneux à faune bathonienne. } \\
\text { Calcaire fin, gris clair, à débris de coraux et brachiopodes. }\end{array}$ \\
\hline & Inf. & Lias & $j_{s}$ & $\begin{array}{l}\text { Grès à lignite associé à du calcaire à petites gastéropodes } \\
\text { lacustres. } \\
\text { Dolomie compacte ou friable. Calcaire gris, partiellement } \\
\text { dolomitisé. Dolomie et intercalations marneuse }\end{array}$ \\
\hline
\end{tabular}


TABLEAUII Description des formations géologiques de l'ère tertiaire. Texte anglais.

\begin{tabular}{|c|c|c|c|c|}
\hline \multirow{3}{*}{$\begin{array}{l}N \\
\text { E } \\
\text { O } \\
\text { G } \\
\text { E } \\
N \\
\text { E }\end{array}$} & Pliocène & Plaisancien & $\mathrm{p}$ & $\begin{array}{l}\text { Marno-calcaires gréseux et marne crayeuse. Calcaire gréseux passant à un } \\
\text { calcaire oolithique poreux. Argile bleue et banc de calcaires détritiques. } \\
\text { Conglomérat et grès jaune avec lentilles de tuf calcaire. }\end{array}$ \\
\hline & \multirow[b]{2}{*}{ Miocène } & Vindobonien & $\mathrm{m}_{3}$ & $\begin{array}{l}\text { Poudingue à galets crétacés et jurassiques. Marne sableuse jaunâtre. } \\
\text { Argiles bleues plaisanciennes. }\end{array}$ \\
\hline & & Moy. & $m_{2}$ & $\begin{array}{l}\text { Conglomérats à galets jurassiques et crétacés. } \\
\text { Calcaire marneux et sableux. }\end{array}$ \\
\hline \multirow{3}{*}{$\begin{array}{l}\mathrm{N} \\
\mathrm{U} \\
\mathrm{M} \\
\mathrm{M} \\
\mathrm{U} \\
\mathrm{L} \\
\mathrm{I} \\
\mathrm{T} \\
\mathrm{I} \\
\mathrm{Q} \\
\mathrm{U} \\
\mathrm{U}\end{array}$} & \multicolumn{2}{|l|}{ Oligocène } & $e_{4}$ & \\
\hline & \multirow[b]{2}{*}{ Éocène } & Priabonian & $e_{3}$ & Gros bancs de calcaire marmoréen bréchique. \\
\hline & & $\begin{array}{l}\text { Lutétien } \\
\text { Ypresien }\end{array}$ & $\begin{array}{l}e_{2} \\
e_{4}\end{array}$ & $\begin{array}{l}\text { Marne calcaire et rognons de silex. } \\
\text { Alternance de craie et de bancs de calcaires tendres. } \\
\text { Calcaire franc, récifal, blancs et brèche à gros élèments. } \\
\text { Brèches á gros blocs calcaires noyés dans une marne contenant localement } \\
\text { beaucoup de glauconie. } \\
\text { Alternance de marno-calcaires et de fins bancs de calcaires. } \\
\text { Marno-calcaires à fins lits de silex blond. }\end{array}$ \\
\hline
\end{tabular}

face. Ces formations sont suivies en profondeur par les autres formations (Miocène et Crétacé, en particulier) qui font l'objet de cet article.

\section{3}

\section{Caractéristiques géotechniques des formations géologiques}

Nous décrirons dans cette partie les spécificités géotechniques des différentes formations selon une nouvelle classification proposée. Cependant, des caractéristiques communes aux formations géologiques diversifiées méritent d'être soulignées.

\section{1}

\section{Caractéristiques d'ordre général}

\section{3ilit \\ Paramètres mécaniques}

Mis à part quelques sols mous et organiques qui se trouvent au bord des rivières et les sols gonflants dans certains bassins sédimentaires, la nature raide ou très dense est une caractéristique générale du sol au Liban. Ces sols alternent avec les roches dures et compactes. De ce fait, les valeurs des paramètres de résistance sont souvent sous-estimées. D'autre part les paramètres de déformations (élastiques et anélastiques) sont de peu d'importance, et pour cela très mal connus. Il est rare de trouver publiées des valeurs fiables issues d'une campagne de caractérisation précise.

\section{3h? \\ Conditions hydrauliques}

Les milieux aquifères sont rencontrés dans des étendues de plusieurs formes. Outre la nappe phréatique de la côte méditerranéenne ; des milieux aquifères locaux peuvent être rencontrés à des altitudes supérieures. C'est le cas de la partie nord de Beyrouth (altitude supérieure à $100 \mathrm{~m}$ ) où des nappes sont confi-
Diversification des formations géologiques à Beyrouth. 
nées dans des formations marneuses étanches. Ces formations sont sujettes à différentes pressions piézométriques. En revanche, les coefficients de perméabilité sont trop petits. De ce fait, le problème de rabattement de la nappe peut être résolu par une simple procédure de pompage. Éventuellement dans ce cas, sans se préoccuper du dimensionnement, les ingénieurs placent leurs installations hydrauliques (pompes, conduites...) suivant les conditions géométriques et pratiques du projet.

\section{3hitiz}

\section{Hétérogénéité}

C'est la caractéristique omniprésente dans presque toute excavation dans les formations tertiaires et même quaternaires. Les blocs pierreux et les cailloux se trouvant dans une matrice sableuse ou argileuse sont un exemple typique. Même dans les sols quaternaires, la décalcification des sables cimentés crée une hétérogénéité. Cette décalcification est due à la fluctuation saisonnière de la nappe phréatique.

L'hétérogénéité met les ingénieurs devant la difficulté de la représentativité des échantillons caractérisés au laboratoire, mais aussi devant un dilemme : choisir entre l'approche de la mécanique des sols classique ou la mécanique des roches.

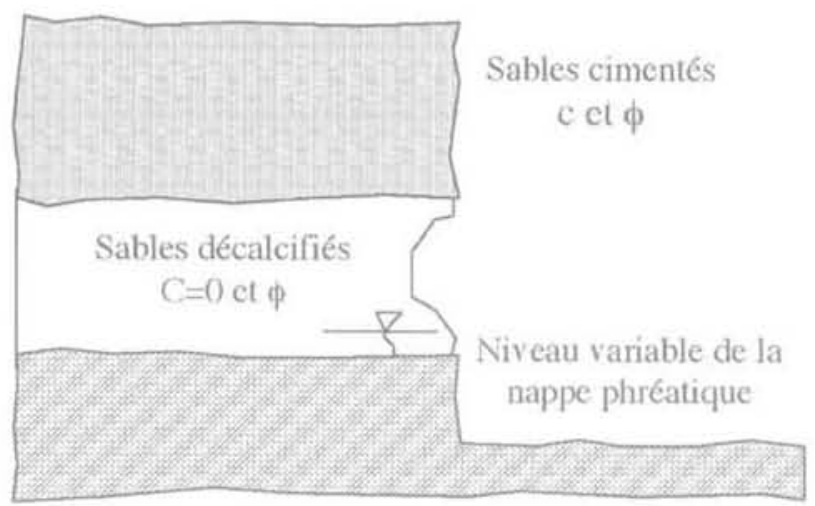

FIG.3 Hétérogénéité dans les formations quaternaires due à la décalcification des sables cimentés. simple des échantillons intacts dépasse les $50 \mathrm{MPa}$. Toutefois, des fractures verticales remplies d'argile sont à l'origine de quelques altérations météoriques des couches relativement profondes. Le module d'élasticité de ces géomatériaux étant souvent supérieur à $40 \mathrm{GPa}$, leur déformabilité n'entre pas en considération dans le dimensionnement des ouvrages. Son comportement anélastique est négligé et mal connu.

Le caractère sédimentaire de la genèse de ces formations, ainsi que les fines intercalations marneuses, ne réduisent pas leur indice de qualité ; leur RQD étant souvent supérieur à $75 \%$. La présence de quelques nodules siliceux réduit la longueur des échantillons prélevés lors des campagnes de reconnaissance de sols et laisse aux ingénieurs des impressions erronées sur la grandeur des bancs du massif. Ces inclusions étrangères et les altérations ne réduisent pas la tenue mécanique des massifs. Par contre, elles créent des plans de faiblesses qui facilitent énormément les travaux d'excavations. L'orientation des joints étant parfois défavorable dans les excavations, cela provoque des problèmes de glissement (Kazan, 2002). En cas de nécessité, le soutènement de ces massifs peut se faire ponctuellement.

Le coefficient de perméabilité de ces formations rocheuses étant inférieur à $10^{-12} \mathrm{~m} / \mathrm{s}$, une éventuelle infiltration dans les massifs correspondants sera due aux fractures existantes et aux joints.

Ces formations sont souvent surincombées par des formations quaternaires. Les travaux de soutènement provisoire des formations superficielles s'arrêtent au niveau jurassique, La figure 4 schématise les procédures proposées pour le soutènement. Les parties supérieures sont traitées par des procédures de soutènements «lourdes $»$. Un écran vertical constitué de pieux espacés de sections circulaires est le mode le plus utilisé. La stabilité de cet écran est assurée par des tirants actifs forés suivant une légère inclinaison. Quant aux couches jurassiques inférieures, un traitement ponctuel par des clous passifs est éventuellement effectué, dans le cas où des fractures verticales sont présentes.

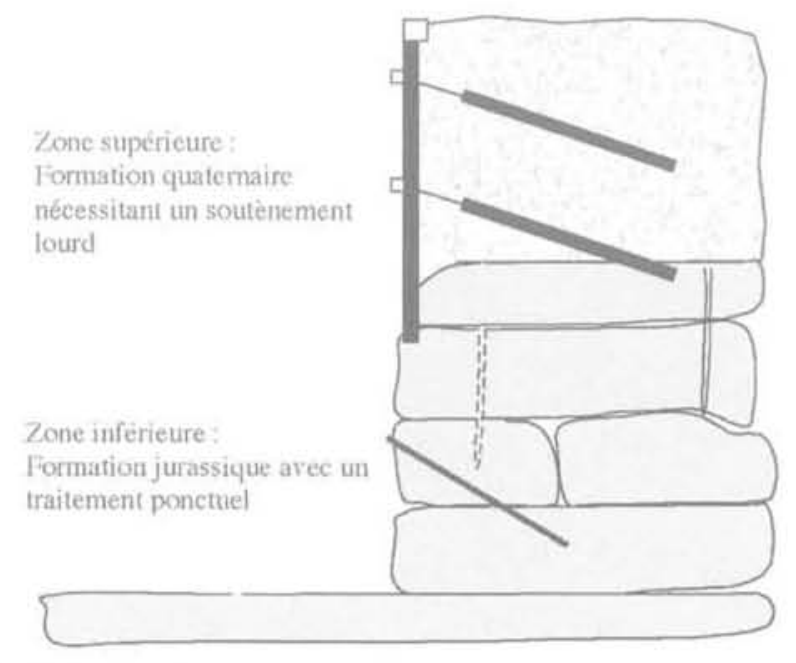

Schématisation des soutènements dans les formations jurassiques.

Il s'agit de formations rocheuses calcaires ou marneuses très résistantes. La résistance à la compression 


\section{Base du Crétacé}

Ces formations peuvent être classées, selon leurs compositions chimiques et minéralogiques, en deux parties : grès et marne. Toutefois, elles ont des caractéristiques communes qui conduisent à préconiser le même mode de soutènement.

Bien que les valeurs de leurs paramètres mécaniques soient plus petites que celles des formations jurassiques, ces valeurs restent bonnes pour les ouvrages de terrassement. Cependant, des avantages spécifiques sont accordés à ses formations :

- pas d'hétérogénéité considérable dans ces massifs. L'isotropie permet de préconiser des procédures d'exécutions simplifiées et répétitives, ce qui a un intérêt économique;

- absence de fractures et de físsures, ce qui écarte tous les problèmes de discontinuités :

- le matériau est suffisamment cohésif pour tenir sans soutènement à très court terme. Il est en mème temps suffisamment détritique et friable pour les travaux de forages.

Le coefficient de perméabilité de cette formation est inférieur à $10^{-9} \mathrm{~m} / \mathrm{s}$ pour le grès et $10^{-11} \mathrm{~m} / \mathrm{s}$ pour les marnes. Des milieux aquifères locaux sont omniprésents dans les milieux marneux. Le rabattement des nappes se fait par de simples procédures de pompage.

\section{4}

\section{Crétacé (moyen et supérieur) et Éocène}

Suivant la chronologie géologique, c'est à partir de ces formations que les travaux de soutènement deviennent indispensables dans les excavations profondes. II s'agit globalement d'une formation de calcaire tendre et de marne raide. La résistance à la compression simple des échantillons marneux prélevés est de l'ordre de quelques $\mathrm{MPa}$. Le recouvrement solide prélevé lors des carottages est souvent inférieur à $60 \%$, alors que le RQD est souvent de l'ordre de $30 \%$. Ces formations contiennent fréquemment des bancs de calcaire résistant semblables aux formations géologiques.

Ce sont ces formations qui rendent les ingénieurs perplexes. Les éléments calcaireux créent des difficultés aux niveaux des procédures d'exécution.

\section{5}

\section{Néogène et Quaternaire alluvionnaire}

Les formations de l'époque Néogène sont les plus déconcertantes. Faut-il les traiter comme un massif rocheux ou plutôt comme un sol ? Les poudingues et les brèches forment une grande partie de cette formation. Outre le problème d'hétérogénéité évoqué au début de cette partie, nous citons les difficultés suivantes :

- les joints entre les blocs sont aléatoirement affaiblis par des conditions extérieures ou intérieures (altération, matériau de remplissage, infiltration d'eau...); - la présence des failles, verticales ou obliques, est fréquente dans ce type de formations. Ces failles les séparent des massifs quaternaires plus homogènes (grès, sable ou autres) :
- dans le cas oủ une matrice argileuse englobe les blocs rocheux, le gonflement de l'argile provoque sur l'écran de soutènement une pression difficile à estimer. Cette pression dépend du pourcentage des blocs, lequel varie avec la profondeur. Il est d'ailleurs difficile d'estimer ce pourcentage lors des campagnes de reconnaissance du sol.

\section{6}

\section{Quaternaire}

Ce sont les formations les plus classiques. Elles seront traitées selon l'approche classique de la mécanique des sols. Elles ne font pas l'objet de remarques particulières.

\section{4 \\ Réflexions sur l'état actuel des pratiques géotechniques locales}

Les différents projets réalisés par plusieurs compagnies ont été exécutés par des techniques variées provenant de différents pays et dont certaines sont universellement adoptées. Il est ainsi intéressant de voir dans une surface relativement restreinte (quelques kilomètres carrés), plusieurs techniques vérifiées et validées par différentes approches de calcul, exécutées l'une à côté de l'autre et justifiées par des raisons géologiques ou économiques. Parmi les rares publications de cette expérience, nous citons (Esta, 1999). Toutefois, un certain recul est nécessaire pour faire ressortir les difficultés et souligner les imprécisions dans les pratiques locales.

\section{1}

\section{Reconnaissance et caractérisation des sols et essais in situ}

Les moyens et les méthodes de reconnaissance des sols employés dans le monde sont variés et très nombreux. Ils dépendent a priori de la nature géologique des sols, mais ils sont pratiquement liés à l'histoire du développement du métier dans chaque pays. Au Liban, la plupart de ces moyens de reconnaissance sont employés. C'est ainsi que nous voyons, à titre d'exemple, les essais pressiométriques de la France se dérouler conjointement avec les essais standards de pénétration des États-Unis (Standard Penetration Test, SPT).

Les géotechniciens s'efforcent d'employer la méthode adéquate à leurs terrains, mais non sans difficulté. Illustrons ces propos par les deux cas suivants :

- les très grandes valeurs enregistrées lors des essais in situ de pénétration, dues à l'hétérogénéité des massifs gréseux par exemple, rendent les résultats des essais pratiquement inexploitables. Les ingénieurs essayent « d'atténuer » les valeurs enregistrées pour rester dans le domaine raisonnable et pour ne pas se montrer trop optimistes. Dans ce cas, l'essai lui-même sert seulement pour la forme. Nous sommes donc ame- 
nés maintenant à mener des études empiriques pour établir une corrélation locale avec les vrais paramètres mécaniques des sols ;

- une large gamme de massifs peut être classée à la fois comme roche tendre ou sols raides et denses, C'est le cas du grès cimenté et de la craie dans les formations tertiaires. Cette complexité laisse l'ingénieur dans l'embarras devant l'approche mécanique à prendre en compte pour dimensionner la structure.

\section{2}

\section{Méthodes de validations des structures géotechniques}

Au Liban, la validation d'une étude d'un sol-structure se vérifie souvent, voir exclusivement, par la vérification a posteriori de sa stabilité vis-à-vis de la rupture. Cette façon de valider est vicieuse, parce que la stabilité d'une structure n'est due ni à un bon calcul de structure, ni au dimensionnement de ses éléments, ni à la bonne exécution de ses différentes parties. Le fait que les travaux de terrassement s'achevent sans un problème de rupture ne valide pas la bonne conception et le dimensionnement optimal du projet.

La validation de l'étude exige le suivi de l'interaction sol-structures et la vérification cle ce comportement conformément aux études faites à ce stade. Cela nécessite des mesures de déplacement, extensométriques ou topographiques, des mesures inclinométriques et, éventuellement, des mesures de contraintes de relaxation.

\section{5}

\section{Nouvelle classification géotechnique et modes de soutènement correspondants}

En tenant compte de l'analyse globale de tous les problèmes énumérés auparavant et des caractéristiques des formations géologiques, nous proposons pour les modes de soutènement la classification du tableau III.

Cette nouvelle classification regroupe les périodes géologiques suivant le mode de soutènements à préconiser lors des travaux géotechniques de soutènement. Les modes de soutènements dans cette classification varient entre le $\alpha$ sans soutènement $)$ et le « soutènement lourd x. Les géotechniciens décideront, selon le contexte, le type précis à préconiser suivant le mode suggéré ici. Cette nouvelle classification pourrait servir comme guide pour les campagnes de reconnaissance de sols en accentuant les paramètres et les facteurs particuliers à étudier pour chaque formation géologique correspondante.

L'auteur ne prétend pas que cette classification est exhaustive. Il reconnait que les limites entre les différentes catégories de cette classification peuvent ne pas être bien définies, et son sujettes à des modifications, voire rectification. Toutefois, elle permettra aux ingénieurs géotechniciens de s'approcher du contexte géologique de leurs projets par une vision géologique plus large.

TABLEAUIII Nouvelle classification géotechnique des formations géologiques libanaises. Texte anglais.

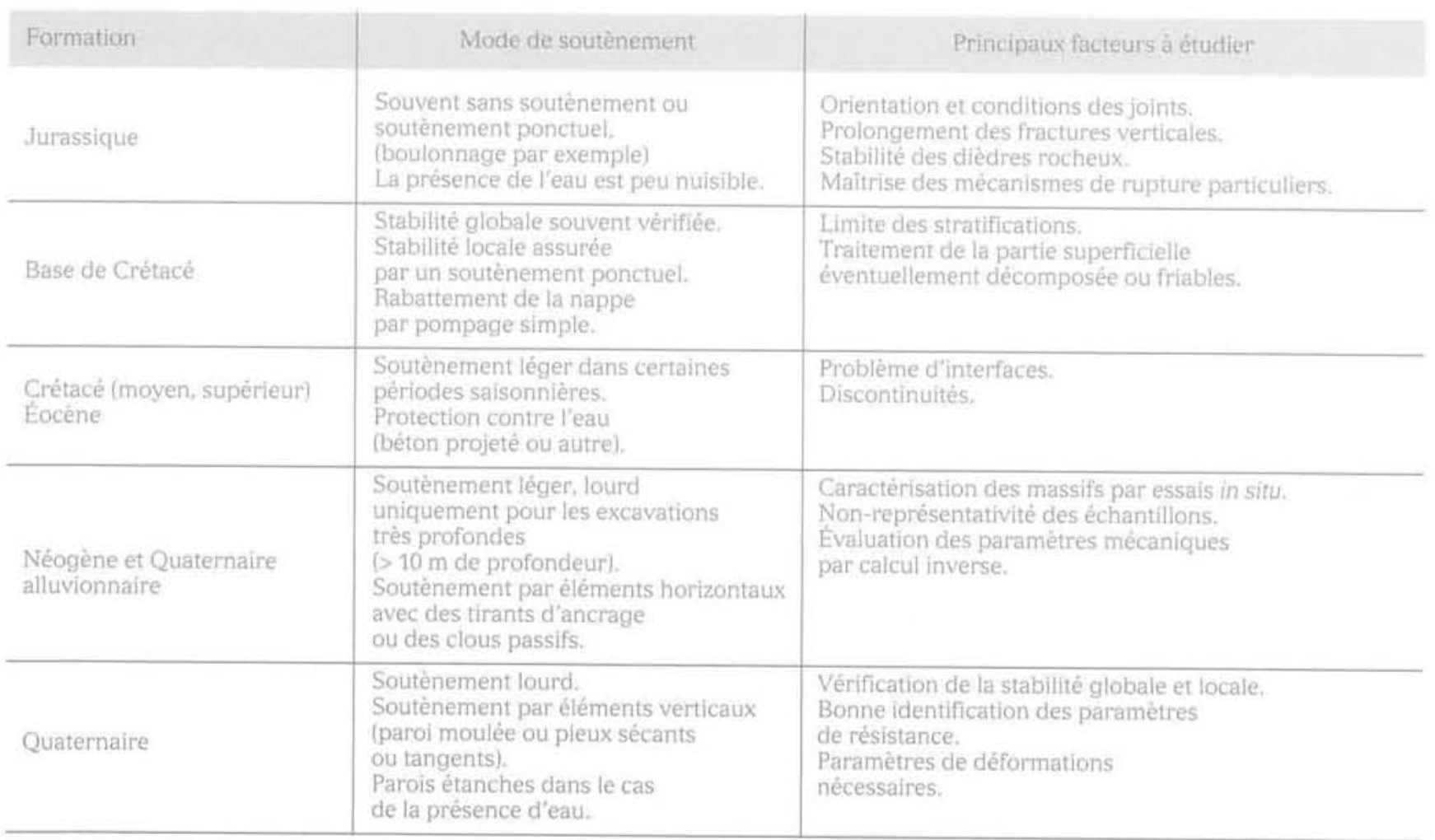




\section{Conclusion et perspectives}

Cet article a présenté les principaux traits des différentes formations géologiques au Liban. Les caractéristiques mécaniques communes de ces formations ont été mises en évidence et exposées.

Les réflexions sur les approches adoptées en travaux géotechniques à Beyrouth ont montré les difficultés qui accompagnent les procédures de caractérisation des sols. Les critiques des erreurs courantes dans l'interprétation des résultats ont souligné la nécessité de trouver des corrélations locales entre ces résultats et les vrais paramètres.
Une nouvelle classification géotechnique a pu être proposée pour les différentes formations géologiques au Liban. Elle accentue indirectement l'utilité des anciens travaux des géologues. Bien que sommaire, cette classification peut servir actuellement comme référence pour les avant-projets géotechniques et pour les conceptions préliminaires des campagnes de reconnaissance des sols. Elle doit être affinée par d'autres analyses ultérieures, basées sur des cas documentés et publiés. Nous espérons que le travail fourni dans cet article, concernant les formations géologiques au Liban, permettra d'ouvrir le débat entre ingénieurs qui interviennent dans cette partie du monde.

\section{Bibliographie}

Abd-el-Al t, - « L'originalité de l'écoulement dans les massifs calcaires libano syriens $₫ 2$. Congrès technique international, Le Caire, 1949

Dubertret L. - $\alpha$ Carte géologique au $1 / 50000$ du Liban » diffusée par le ministère des Ressources hydrauliques. 1951.

Esta J.-B, - « Paroi clouèe et tirantée à Beyrouth $\%$. Revue française de géotechnique $n^{\circ} 89,4$ trimestre 1999.
Farras O. - "Aus dem Orient. Geologische Beobachtungen am Libanon n. Wurttemb. Naturw, Yahreshefte, Stuttgart, 1878, p. 257-392, pl. III-VIII.

Kazan Y. - - Mẻcanisme de rupture dû́ à un problème d'interface, cas réel interprétation et recommandations $x$. Journées nationales de géotechnique et de géologie de l'ingénieur, JNGG-2002, Nancy, France, 2002.
Khawlie M.R., Ghannam J. - $\alpha$ Land suftability and geotechnical studies for the development of greater Beirut Area - Lebanon p. Geological Society of Hong Kong Bulletin $n^{2} 3$, October 1987.

Kober L - "Geologische Forschungen in Vorderasien. Zur Tektonik des Libanon " Denkschr. math. Naturw. KI. K. Ak. Wiss., Wien, 1915, p. 419-424. 